\title{
Aurapten, a coumarin with growth inhibition against Leishmania major promastigotes
}

H.B. Napolitano ${ }^{1}$, M. Silva ${ }^{1}$, J. Ellena ${ }^{1}$, B.D.G. Rodrigues ${ }^{1}$, A.L.C. Almeida², P.C. Vieira², G. Oliva ${ }^{1}$ and O.H. Thiemann ${ }^{1}$

\author{
'Laboratório de Proteína, Cristalografia e Biologia Estructural, \\ Instituto de Física de São Carlos, Universidade de São Paulo, São Carlos, \\ SP, Brasil \\ ${ }^{2}$ Departamento de Química, Universidade Federal de São Carlos, São Carlos, \\ SP, Brasil
}

\begin{abstract}
Correspondence
O.H. Thiemann

Laboratório de Proteína,

Cristalografia e Biologia Estructural

Instituto de Física de São Carlos

USP

Av. Trabalhador Sãocarlense, 400

13566-590 São Carlos, SP

Brasil

Fax: +55-16-273-9881

E-mail: thiemann@if.sc.usp.br

Research supported in part by FAPESP (No. 99/02874-9 to O.H. Thiemann). H.B. Napolitano and M. Silva are recipients of FAPESP fellowships.
\end{abstract}

Received March 22, 2004 Accepted August 24, 2004

\begin{abstract}
Several natural compounds have been identified for the treatment of leishmaniasis. Among them are some alkaloids, chalcones, lactones, tetralones, and saponins. The new compound reported here, 7geranyloxycoumarin, called aurapten, belongs to the chemical class of the coumarins and has a molecular weight of 298.37. The compund was extracted from the Rutaceae species Esenbeckia febrifuga and was purified from a hexane extract starting from $407.7 \mathrm{~g}$ of dried leaves and followed by four silica gel chromatographic fractionation steps using different solvents as the mobile phase. The resulting compound $(47 \mathrm{mg}$ ) of shows significant growth inhibition with an $\mathrm{LD}_{50}$ of $30 \mu \mathrm{M}$ against the tropical parasite Leishmania major, which causes severe clinical manifestations in humans and is endemic in the tropical and subtropical regions. In the present study, we investigated the atomic structure of aurapten in order to determine the existence of common structural motifs that might be related to other coumarins and potentially to other identified inhibitors of Leishmania growth and viability. This compound has a comparable inhibitory activity of other isolated molecules. The aurapten is a planar molecule constituted of an aromatic system with electron delocalization. A hydrophobic side chain consisting of ten carbon atoms with two double bonds and negative density has been identified and may be relevant for further compound synthesis.
\end{abstract}

\section{Introduction}

Leishmaniasis is a disease caused by a protozoan parasite of the order Kinetoplastida. According to the World Health Organization (1), 12 million infected people exist in 88 countries, with approximately 350 million people being at risk. In view of the lack of safe drugs and the serious secondary effects caused by available chemotherapy (2), there is a need
Key words

- Coumarin

- Aurapten

- X-rays

- Leishmania

- Inhibitor (7-geranyloxycoumarin) for new drugs for the treatment of leishmaniasis infections. Therefore, the discovery of novel classes of inhibitors can be an important step which contributes to overcoming the drug resistance of Leishmania (3).

The use of phytotherapy to treat human diseases has its roots in pre-historical times and most of the effective drugs currently available are obtained from plants (4). The biodiversity existing in Brazilian plants is a poten- 
tial source of many new bioactive molecules to be studied and explored (5).

In a search for new bioactive substances potentially useful against leishmaniasis, we used Leishmania major Friedlin (WHO MHOM/IL/80/Friedlin) cells as a model system to screen small molecule compounds obtained from Brazilian plants. The screening was done using an in vivo culture assay in the presence or absence of the purified compound and the parasite growth rate was monitored microscopically. This approach permitted us to identify a new compound which was subsequently shown to be 7-geranyloxycoumarin (aurapten). The compound belongs to the class of coumarins and shows significant growth inhibition of $L$. major promastigotes at concentrations of micrograms per $\mathrm{ml}$.

\section{Material and Methods}

\section{Extraction of the plant inhibitor}

The leaves of Esenbeckia febrifuga (Rutaceae) were collected in Piracicaba, SP, Brazil, by Prof. Ricardo Ribeiro Rodrigues (Escola Superior de Agricultura Luiz de Queiroz, ESALQ, Code No. 84295) in February 2000 and extracted according to the procedure illustrated in Figure 1 and described below.

Ground leaves $(407.7 \mathrm{~g}$ ) were submitted to a first extraction step with 4.5 liters hexane, resulting in $9.5 \mathrm{~g}$ of extract after the solvent was removed. The extract was fractionated by silica gel chromatography through a $30 \mathrm{~cm}$ high column measuring $5 \mathrm{~cm}$ in diameter using a hexane/ethyl acetate (100:0, 9:1, 8:2, 7:3, $1: 1,0: 100)$ and an ethyl acetate/methanol (100:0, 9:1, 7.5:2.5, 6:4, 1:1, 0:100) gradient system. From this purification, 30 fractions of $25 \mathrm{ml}$ each were collected. Fraction 14 (hexane:ethyl acetate, 8:2) was further fractionated on a 3 x $26 \mathrm{~cm}$ silica gel column using a gradient system with hexane, methylene chloride and methanol (hexane, 100\%; hexane/ methylene chloride, 100:0,90:10, 80:20,70:30, 1:1; methylene chloride/methanol, 100:0,
90:10, 70:30, 1:1, 0:100). The 74 fractions ( 25 $\mathrm{ml}$ each) were collected and, based on thinlayer chromatography (TLC) on silica gel (0.25-mm thick silica gel, Merck PF254(Darmstadt, Germany), hexane/methylene chloride/ acetone, 3:1:0.5), and detected with vanillic acid, UV light at 254 and $360 \mathrm{~nm}$ and Dragendorff reagent, eight fractions were pooled. Fraction four was submitted to chromatographic separation on a $2 \times 31 \mathrm{~cm}$ silica gel column eluted isocratically with hexane, methylene chloride and acetone (3:1:0.5). This step resulted in 87 fractions $(25 \mathrm{ml}$ each), twelve of which were pooled after TLC analysis as above. Fraction two was submitted to chromatographic separation on a $1 \times 20 \mathrm{~cm}$ silica gel column using methylene chloride as the mobile phase and resulted in 24 fractions ( $25 \mathrm{ml}$ each), four of which were pooled after TLC analysis. Fraction four resulted in an amorphous yellow solid (47 mg), which is 7geranyloxycoumarin (aurapten), according to NMR ${ }^{1} \mathrm{H},{ }^{13} \mathrm{C}$ spectra (named after the standard chemical nomenclature according to IUPAC rules) and GC/MS, that was crystallized by vapor diffusion, at room temperature, from hexane/methylene chloride (2:1). The purity of the compound was confirmed by TLC (0.25-mm thick silica gel), NMR and GC/MS.

\section{Parasite assays}

Stock solutions of aurapten were prepared in dimethyl sulfoxide (DMSO) at $5.0 \mathrm{mg} / \mathrm{ml}$. Further dilutions of aurapten were made directly in the $L$. major culture medium immediately before use. In all experiments the final concentration of DMSO was less than $0.5 \%$ $(\mathrm{v} / \mathrm{v})$, a concentration that does not affect parasite growth rate, mobility or morphology (6).

L. major Friedlin promastigotes were grown to a concentration of $1.5 \times 10^{4}$ cells $/ \mathrm{ml}$ in M199 (Gibco Laboratories, New York, NY, USA) supplemented with $10 \%$ fetal bovine serum, $10 \mathrm{mM}$ adenine, $10 \mathrm{mg} / \mathrm{ml}$ penicillin, $10 \mathrm{mg} / \mathrm{ml}$ streptomycin, $0.25 \%$ hemin chlo- 
ride, $0.1 \%$ biotin, and $1.0 \mathrm{M}$ HEPES, $\mathrm{pH}$ 7.5. The parasite cultures were prepared with or without aurapten at final concentrations of 2.0, 5.0, 7.0, 10.0, and $15.0 \mu \mathrm{g} / \mathrm{ml}$. The effect of aurapten on parasite growth was monitored by counting in triplicate in a Nebauer chamber after 48 -h incubation at $26^{\circ} \mathrm{C}$.

\section{Crystallographic characterization of aurapten}

Aurapten was crystallized from hexane/ methylene chloride $(2: 1)$ by vapor diffusion at room temperature. The typical crystal was yellow and was needle shaped. X-ray diffraction was carried out using a Nonius KappaCCD diffractometer at room temperature. The structure was solved by direct methods using the SHELXL97 software (7) and was refined anisotropically with full-matrix least-squares on $F^{2}$ using SHELXL97 (7). The hydrogen atoms were placed at the calculated position, except for those involved in H-bonds, found on difference maps and further refined. Final indices were: $\mathrm{R}_{1}\left(F_{\mathrm{o}}\right)=0.0895$ and $\mathrm{wR}_{2}\left(F^{2}\right)=0.2723$ for 165 refined parameters. The crystallographic parameters for $\mathrm{C}_{19} \mathrm{H}_{22} \mathrm{O}_{3}$ are: molecular weight $=298.37$, orthorhombic, space group Pnma [No. 62], $\mathrm{Z}=4, a=6.557$ (1) $\AA$, $b=$ 7.117 (2) $\AA, c=35.650$ (2) $\AA, V=1664.0$ (9) $\AA^{3}, d_{c}=1.191 \mathrm{Mg} \cdot \mathrm{m}^{-3}, \lambda($ Mo K $\alpha)=0.71073$ $\AA, \mu=0.104 \mathrm{~mm}^{-1}, 3205$ measured reflections, 1616 unique $\left(R_{\text {int }}=0.0301\right), 1579$ of which were considered for calculation purposes as observed with an I $\geq 2 \sigma$ (I). The crystallographic data were deposited in the Cambridge Crystallographic Data Centre (deposit@ccdc. camac.uk) under accession number 232201.

\section{Results and Discussion}

We investigated and characterized the antiproliferative activity of the coumarin (7geranyloxycoumarin), or aurapten, with a molecular weight of 298.37 against $L$. major Friedlin promastigotes in axenic cultures. As shown in Figure 2, L. major promastigote growth was significantly inhibited by aurapten, with an $\mathrm{LD}_{50}$ of $30 \mu \mathrm{M}$. This value represents a significant inhibitory activity against $L$. $m a$ jor and is similar to those obtained for other inhibitors isolated from other plants (8). The negative control, corresponding to the parasite in the presence of DMSO, the aurapten sol-

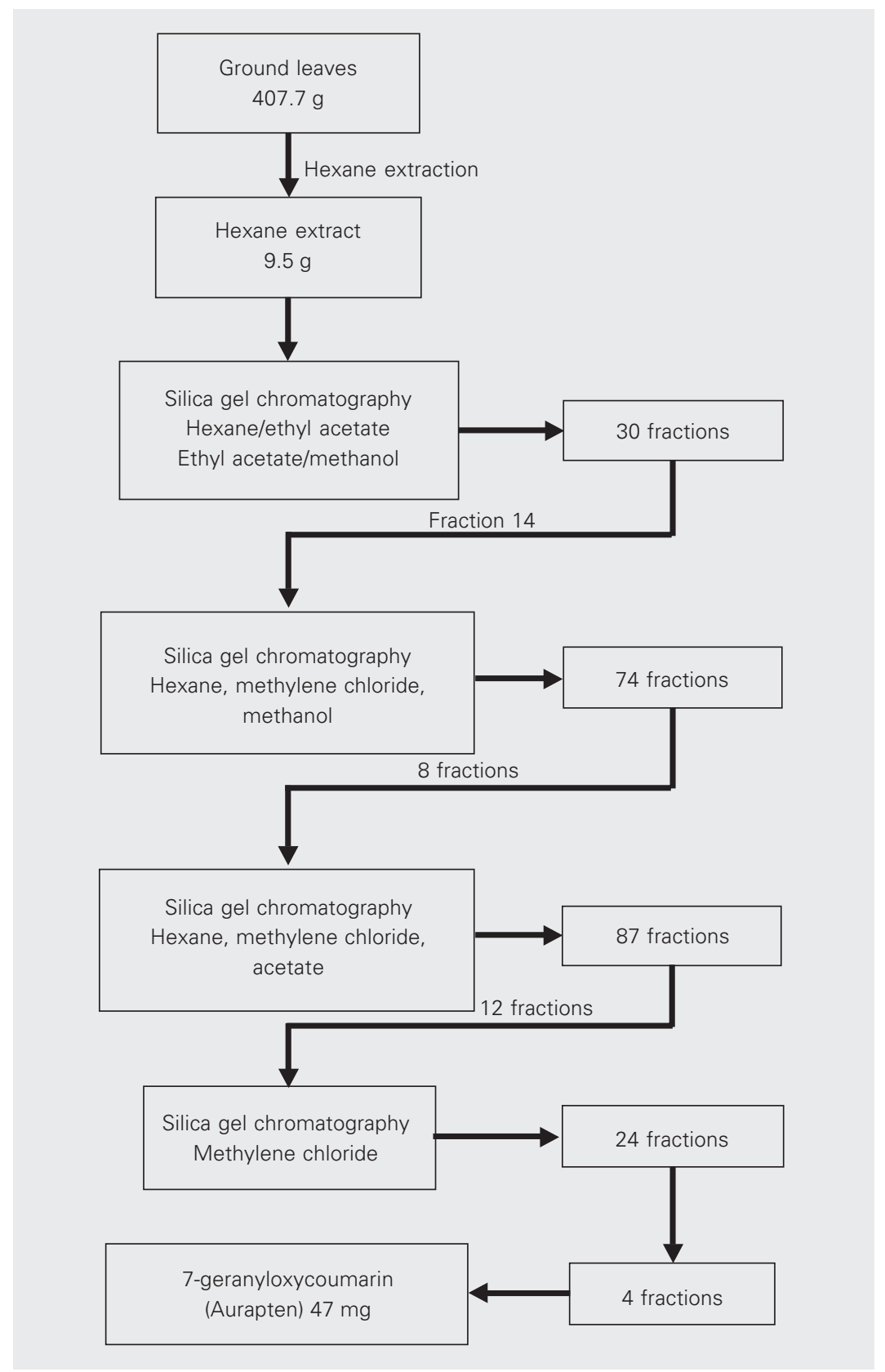

Figure 1. Flow diagram of the aurapten isolation procedure. Individual steps are described in Material and Methods. The solvents used for elution of the chromatography column are listed in the boxes on the left side. Silica gel column effluents were monitored by thin-layer chromatography to select fractions for further purification. 
Figure 2. Antileishmanial activity of aurapten against Leishmania major Friedlin promastigotes. Cells were cultivated in the presence of different concentrations of aurapten and counted after 48 $h$. The height of the bars indicates de percentage of growth inhibition at each concentration compared to the control experiment containing only the aurapten solvent DMSO. The experiments were performed three times independently and each counting was performed in triplicate. Data are reported as means \pm SD.

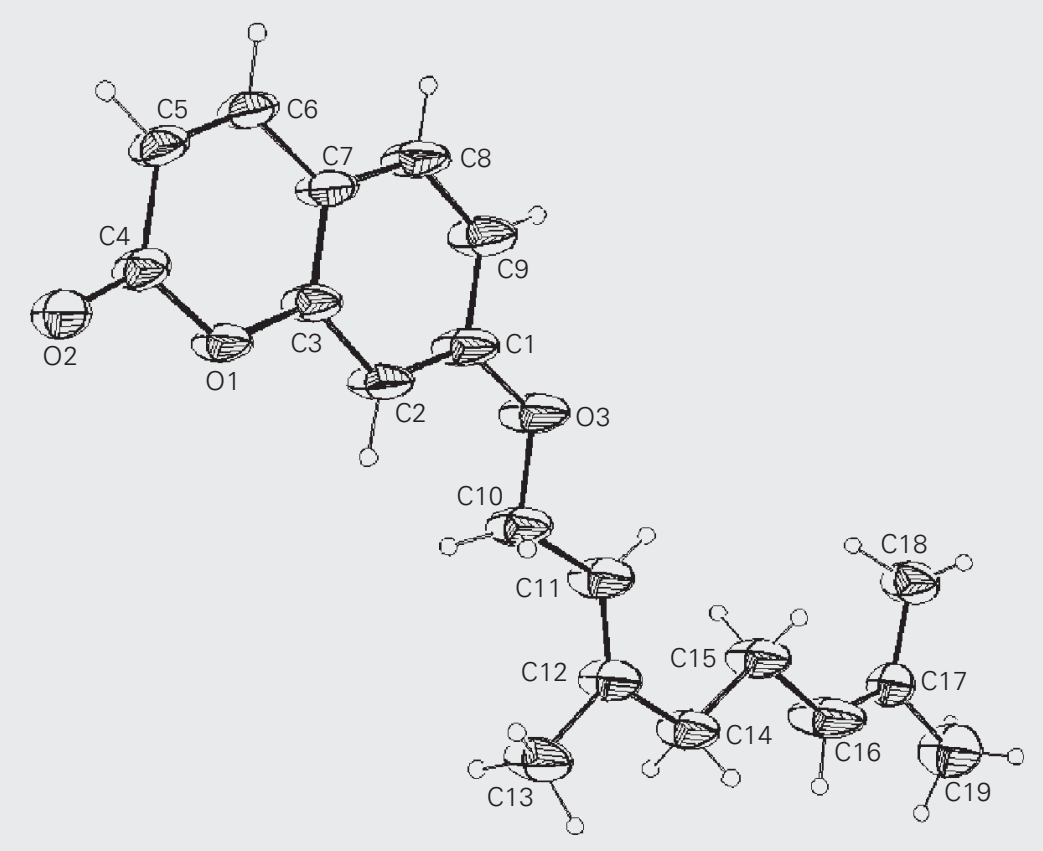

Figure 3. X-ray crystallographic structure of the aurapten. Displacement ellipsoids are shown at the $30 \%$ probability level. Carbon (C) and oxygen (O) atoms are labeled for clarity. vent, showed no detectable inhibition of growth, alteration in cell morphology or motility at the concentrations tested.

Most studies directed at the detection of plant secondary metabolites with leishmanicidal activity have been done using the promastigote form of the parasite because of its easier maintenance under in vitro conditions. However, since the promastigote is not the infective form of the parasite in vertebrate hosts, these evaluations have only a suggestive value of the possible leishmanicidal activity of the compound tested.

There are several active compounds obtained from medicinal plants traditionally used worldwide for the treatment of leishmaniasis. Among these active molecules, described in the recent literature are quinoline alkaloids, isoquinoline alkaloids, indole alkaloids, terpenes, acetogenins, and lignans (9). Other natural compounds with antileishmanial activity are coumarins, chalcones, lactones, tetralones, and saponins (9). The compound described in the present study belongs to the class of the coumarins and its activity against Leishmania promastigotes is reported here for the first time.

To unambiguously assign the structure of this inhibitor and to gain insight into common structural motifs related to other coumarins and other leishmania inhibitors, its crystal structure was determined, and several structural features emerged. This new information will be valuable for the development of a new anti-
Figure 4. A view of aurapten's packing showing $\mathrm{C}-\mathrm{H}$...O interactions. Symmetry codes (i) and (ii) correspond to $[1+x, y, z]$ and $[0.5+x, y, 1.5-z]$, respectively.

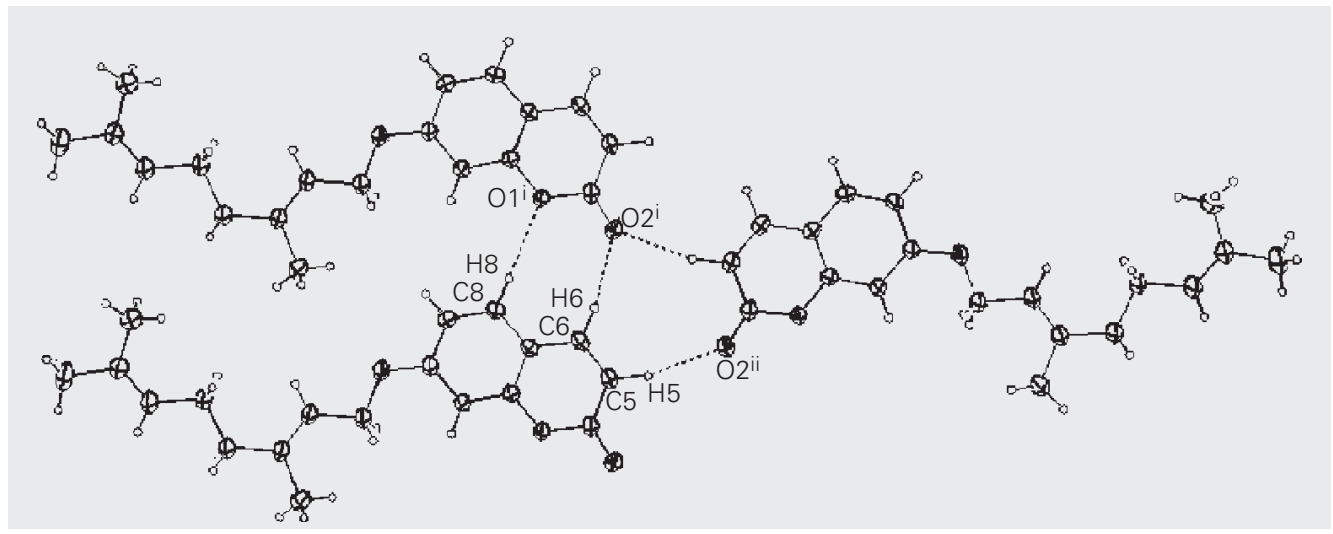


Leishmania drug since resistance to the currently available drugs such as antimonials and amphotericin B has emerged (3).

The refined molecular structure together with the atom-numbering scheme is shown in Figure 3. The molecule is a derivative of cinnamic acid that contains an aromatic system with electron delocalization. There is also a hydrophobic side chain consisting of ten carbon atoms with two double bonds with negative density. All bond distances and angles are close to normal values $(10,11)$ and the molecule is planar, as can be seen in Figure 3. Three double weak intermolecular interactions of type $\mathrm{C}-\mathrm{H}$...O support the molecular packing (12) shown in Figure 4. They are the intermo-

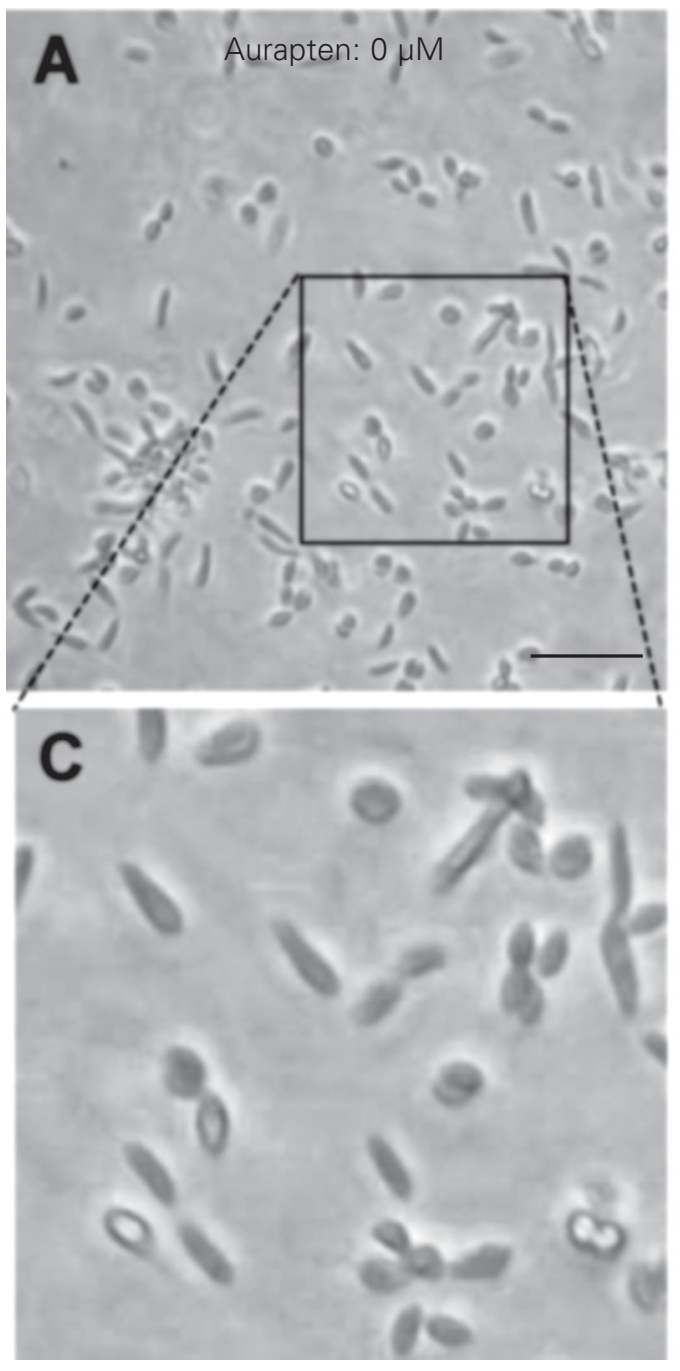

lecular $\mathrm{C} 8-\mathrm{H} 8 . . . \mathrm{O} 1^{\mathrm{i}}$ and $\mathrm{C} 6-\mathrm{H} 6 . . . \mathrm{O} 2^{\mathrm{i}}$ [symmetry code: i) $1+x, y, z]$ hydrogen bonds between two neighbor molecules. The distance and dihedral angles between donor and acceptor are 3.38 (1) $\AA, 180(5)^{\circ}$ and 3.27 (1) $\AA, 172(5)^{\circ}$, respectively. The last of the intermolecular hydrogen bonds $\mathrm{C} 5-\mathrm{H} 5 . . . \mathrm{O} 2^{\mathrm{ii}}$ [symmetry code: ii) $0.5+\mathrm{x}, \mathrm{y}, 1.5-\mathrm{z}]$ linking these two molecules shows 3.34 (1) $\AA$ of distance and $163(3)^{\circ}$ of dihedral angle between donor and acceptor. The planarity shown in the molecular configuration and the intermolecular interactions are consistent with typical structural features observed in anti-Leishmania inhibitors (13-15).

Figure 5 shows the L. major culture in the

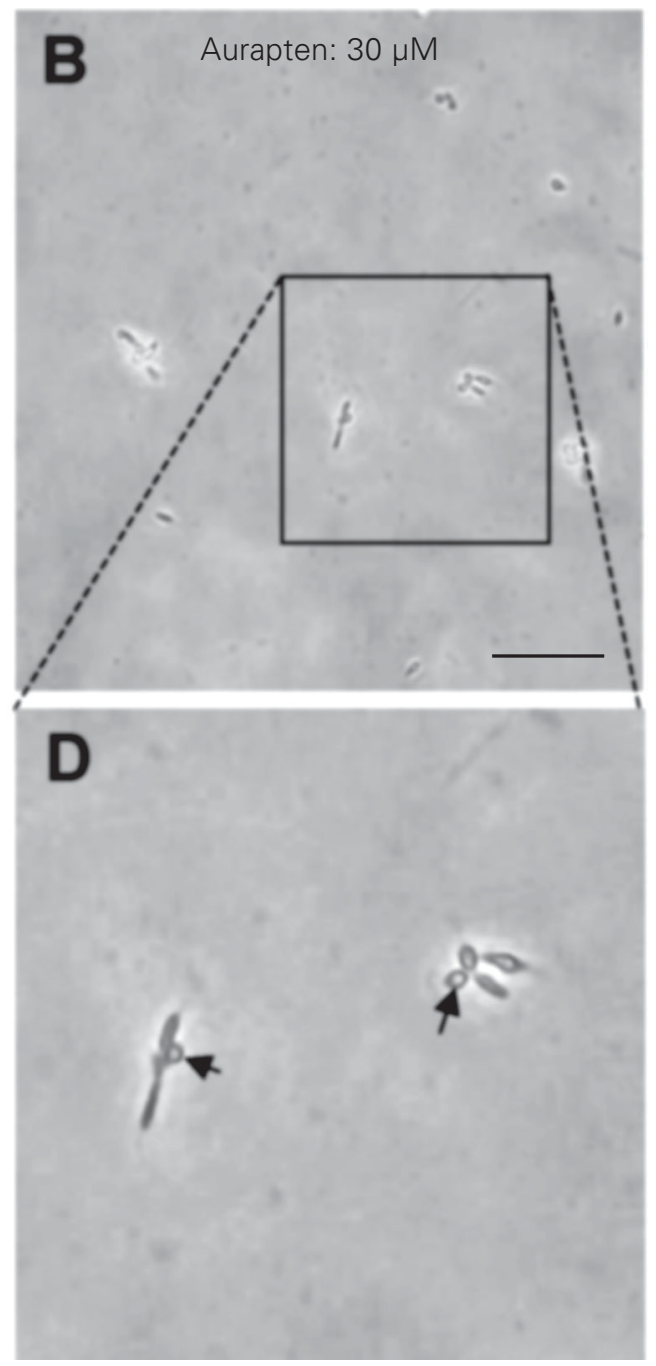

Figure 5. Leishmania major promastigote culture. Promastigotes were cultured in M199 medium supplemented with $10 \%$ fetal bovine serum at $26^{\circ} \mathrm{C}$ and cell viability was estimated by counting in a Neubawer chamber after $48 \mathrm{~h}$ of incubation in the presence or absence of the inhibitor. $A$, Negative control without aurapten in the presence of $0.5 \%$ DMSO. $B$, Condition containing $30 \mu \mathrm{M}$ aurapten and $0.5 \%$ DMSO. $C$ and $D$ are expansions of Figures $A$ and $B$, respectively (indicated by the squares in $A$ and $B$ ), showing the different morphological characteristics of the parasite in the presence of aurapten. Vacuoles are indicated by arrows. Bar $=16.6 \mu \mathrm{m}$. 
presence and absence of the inhibitor. Fortyeight hours after inoculation, a significant decrease in surviving promastigotes was observed in the presence of aurapten (Figure 5B) at a final concentration of $30 \mu \mathrm{M}$. The surviving parasites showed abnormal morphology with the presence of large vacuoles and low flagellum motility. This preliminary evaluation of aurapten's activity using promastigotes yielded interesting results indicating that the compound should be investigated further as a potential anti-Leishmania compound.

\section{Acknowledgments}

We would like to thank the members of the Protein Crystallography and Structural Biology and Biophysics Groups (IFSC, USP) for helpful discussions during the course of the study. We would like to express our gratitude to the organizers of the ACA Summer Course in Small Molecule Crystallography, August 4-13, 2003, for the single crystal diffraction experiment.

\section{References}

1. WHO (1998). World Health Organization, http://www.who.int/tdr/ diseases/leish/diseaseinfo.htm.

2. McGreevy PB \& Marsden PD (1986). Campbell WC \& Rew RS (Editors), Chemotheraphy of Parasitic Diseases. Vol. 1. Plenum Press, New York, 115-127.

3. Croft SL (2001). Monitoring drug resistance in Leishmaniasis. Tropical Medicine and International Health, 6: 899-905.

4. Carvalho PB \& Ferreira EIL (2001). Leishmaniasis phytotherapy. Nature's leadership against an ancient disease. Fitoterapia, 72: 599618.

5. Elisabetsky E \& Shanley P (1994). Ethnopharmacology in the Brazilian Amazon. Pharmacology and Therapeutics, 64: 201-214.

6. Zhai L, Chen M, Blom J, Theander TG, Christensen SB \& Kharazmi A (1999). The antileishmanial activity of novel oxygenated chalcones and their mechanism of action. Journal of Antimicrobial Chemotherapy, 43: 793-803.

7. Sheldrick GM (1997). SHELXL97. Refinement of large small-molecule structures using SHELXL-92. In: Flack HD, Párkányi L \& Simon K (Editors), Crystallographic Computing 6. Oxford University Press, New York.

8. Chan-Bacad MJ \& Pena-Rodrigues LM (2001). Plant natural products with leishmanicidal activity. Natural Product Reports, 18: 674688.
9. Akendengue B, Ngou-Milama E, Laurens A \& Hocquemiller R (1999). Recent advances in the fight against leishmaniasis with natural products. Parasite, 6: 3-8.

10. Farrugia LJ (1997). ORTEP-3 for Windows - a version of ORTEP-III with a Graphical User Interface (GUI). Journal of Applied Crystallography, 30: 565 .

11. Allen FH, Kennard O \& Taylor R (1983). Systematic analysis of structural data as a research technique in organic chemistry. Accounts of Chemical Research, 16: 146-153.

12. Spek AL (2003). Single-crystal structure validation with the program PLATON. Journal of Applied Crystallography, 36: 7-13.

13. Napolitano HB, Silva M, Ellena J, Rocha WC, Vieira PC, Thiemann OH \& Oliva G (2003). Redetermination and comparative structural study of isopimpinellin: a new inhibitor against the Leishmania APRT enzyme. Acta Crystallographica, E59: 01506-01508.

14. Napolitano HB, Silva M, Ellena J, Rocha WC, Vieira PC, Thiemann $\mathrm{OH}$ \& Oliva G (2003). Redetermination of skimmianine: a new inhibitor against the Leishmania APRT enzyme. Acta Crystallographica, E59: 01503-01505.

15. Silva M, Napolitano HB, Ellena J, Rocha WC, Vieira PC, Oliva G \& Thiemann $\mathrm{OH}$ (2003). A potential inhibitor against Leishmania, 3(5,7-dimethoxy-2,2-dimethyl-2H-benzo[b]pyran-6-yl) propionic acid. Acta Crystallographica, E59: 01575-01577. 